\title{
Ulinastatin inhibits NLRP3-induced apoptosis in a PD cell model
}

\author{
Yicheng Lin $^{1}$, Dongsheng Xu ${ }^{2}$, Feng Gao ${ }^{3}$, Xiaolei Zheng ${ }^{4}$ \\ ${ }^{1}$ Cheeloo College of Medicine, Shandong University, Shandong Qianfoshan Hospital, Jinan, China; ${ }^{2}$ Department of Kidney Transplantation, The \\ Second Hospital, Shandong University, Jinan, China; ${ }^{3}$ Key Laboratory for Experimental Teratology of the Ministry of Education and Biomedical \\ Isotope Research Center, School of Basic Medical Sciences, Cheeloo College of Medicine, Shandong University, Jinan, China; ${ }^{4}$ Department of \\ Neurology, The Second Hospital, Cheeloo College of Medicine, Shandong University, Jinan, China \\ Contributions: (I) Conception and design: Y Lin; (II) Administrative support: F Gao; (III) Provision of study materials or patients: D Xu; (IV) \\ Collection and assembly of data: D Xu, F Gao; (V) Data analysis and interpretation: X Zheng; (VI) Manuscript writing: All authors; (VII) Final \\ approval of manuscript: All authors. \\ Correspondence to: Dr. Xiaolei Zheng. Department of Neurology, The Second Hospital, Cheeloo College of Medicine, Shandong University, 247 \\ Beiyuan Road, Jinan 250033, China. Email: nuancheng271447@163.com.
}

Background: Emerging evidence suggests that inflammation induced by the inflammasome plays a crucial role in the course of Parkinson's disease (PD). Ulinastatin (UTI) has shown significant anti-inflammatory effects. However, few studies have examined whether UTI protects neurons through its anti-inflammatory effects in PD. The purpose of this study is to determine whether UTI exerts neuroprotection in a PD cell model and to explore the mechanisms.

Methods: SH-SY5Y cells and nerve growth factor (NGF)-treated PC12 cells were used to establish MPP induced PD cell models. Cells were pre-treated with UTI, then cell viabilities were detected using the MTT assay. Lactate dehydrogenase $(\mathrm{LDH})$ release was detected using the $\mathrm{LDH}$ release assay kit. Inflammatory factors such as IL-1 $\beta$, IL-6, and TNF- $\alpha$ were detected using ELISA. The expression levels of TH, NLRP3, caspase-1, ASC, IL-1 $\beta$, and IL-18 were measured using western blotting, and DA release was detected using HPLC. A NLRP3 activator Nigericin was used to verify the effect of NLRP3 in the neuroprotective mechanism of UTI.

Results: We observed decreased cell viability, increased apoptosis, and increased inflammatory factors such as IL-1 $\beta$, IL-6, and TNF- $\alpha$ in the $\mathrm{MPP}^{+}$induced PD model. We also found decreased DA secretion and TH expression, as well as increased NLRP3, caspase-1, ASC, IL-1 $\alpha$, and IL-18 expression in the MPP induced PD model. These changes were significantly attenuated by UTI pre-treatment in a dose dependent manner. NLRP3 activator Nigericin markedly increased LDH release, accelerated apoptosis, increased inflammation levels and decreased DA secretion and TH expression, suggesting that Nigericin eliminated the neuroprotective effect of UTI on $\mathrm{MPP}^{+}$treated cells.

Conclusions: Our data demonstrated that UTI pre-treatment performed a neuroprotective role in the $\mathrm{MPP}^{+}$induced PD cell models by inhibiting the NLRP3 pathway.

Keywords: Ulinastatin (UTI); Parkinson's disease (PD); NOD-leucine rich repeat and pyrin containing protein 3 (NLRP3); inflammation; neuroprotection

Submitted Mar 29, 2021. Accepted for publication May 13, 2021.

doi: $10.21037 / \mathrm{atm}-21-1882$

View this article at: http://dx.doi.org/10.21037/atm-21-1882 


\section{Introduction}

Parkinson's disease (PD) is the second most common neurodegenerative disease, after Alzheimer disease (1). In addition to classic motor symptoms, such as tremors, rigidity, and slow movement, some non-motor symptoms, such as emotional, cognitive, and olfactory disorders also appear in the early stages of PD. This is accompanied by the apoptosis of dopaminergic neurons, which eventually leads to paralysis and even death, seriously affecting people's quality of life (2-7). However, current treatments for PD, including deep brain stimulation therapy and dopaminergic replacement therapy, have failed to halt or slow down the progression of PD (8). Long-term use of levodopa is associated with severe side effects, including the emergence of drug-induced involuntary movements and motor response fluctuation (9). Finding new potential drugs will provide more options for PD therapy.

As a urinary trypsin inhibitor, ulinastatin (UTI) is a glycoprotein isolated from healthy human urine or synthetically produced. Highly purified UTI has been used clinically for the treatment of acute pancreatitis, chronic pancreatitis, Stevens-Johnson syndrome, and toxic epidermal necrolysis $(10,11)$. Clinical study found that Ulinastatin can reduce postoperative bleeding and cognitive dysfunction in patients undergoing cardiac surgery $(12,13)$. Ulinastatin treatment could alleviate sepsis shock of CLP rats by suppressing the inflammatory responses of the body and strengthening the immune system (14). Additionally, ulinastatin ameliorated the severity of intestinal damage in necrotizing enterocolitis (NEC) by inhibiting apoptosis and suppressing the levels of inflammation cytokines (15). Studies have shown that Ulinastatin can also reduce the level of inflammatory cytokines and inhibit oxidative stress $(16,17)$.

PD pathology is characterized by the loss of dopaminergic neurons in the brain and the accumulation of $\alpha$-synuclein (18). Substantial evidence in autopsies, brain imaging, and biomarkers in bodily fluid have shown that neuroinflammation is a significant feature of PD (19). The activation of glial cells and the release of inflammatory factors have also been significantly observed in PD animal models induced by neurotoxins such as MPTP or 6-OHDA (20). Inflammasomes are polyprotein complexes in the cytosome, including NLRPI, NALP2, NLRC4, AIM2, etc., of which the most studied is the NOD-leucine rich repeat and pyrin containing protein 3 (NLRP3) (21). NLPR3 is a multi-protein complex in cells, mainly composed of receptor molecules (PYD), adaptive proteins (ASCs), and effect proteins (caspase-1) (22). Previous studies have shown that NLRP3 palys a vital role in neurodegenerative diseases $(23,24)$. $\alpha$-synuclein promoted the progression of PD by activating NLRP3 inflammasome in astrocytes (25). The NLRP3 inflammasome is activated and the NLRP3 mRNA expression was up-regulated in peripheral blood mononuclear cells (PBMCs) from PD patients (26). Downregulation of NLRP3 could inhibit inflammation and alleviate MPTP-induced degeneration of DA neurons in MPTP-induced PD mice (27). Hence, studying whether UTI can alleviate PD by regulating NLRP3 might provide theoretical reference for the treatment of PD.

NLRP3 activation is thought to require a twostep process: start-up and activation. The initial process increases the expression of various parts of the inflammasome. There are three models for the progression of NLRP3 inflammasome: reactive oxygen species (ROS) generation by pathogen-associated molecular patterns (PAMPs) and damage associated molecular pattern (DAMPs) (28-32); outflow of potassium ions $\left(\mathrm{K}^{+}\right)$by extracellular Adenosine Triphosphate (ATP) through purinergic $2 \mathrm{X} 7$ receptor (P2X7R) (33), and disruption of phagocytic vesicles including crystals by lysosomes (34). Thioredoxininteracting protein (TXNIP) induces the activation of NLRP3 inflammasome in a ROS-sensitive manner as an endogenous antagonist of thioredoxin (35). NLRP3 agonists such as ATP and nigericin cause mitochondrial damage and decrease of NAD+, thereby promoting the activation of NLRP3 inflammasome (36). The second step is the NLRP3 inflammasome oligomerization, which mediates the proteolysis of pro-caspase- 1 into active caspase-1, thereby activating pro-IL-1 $\beta$ and pro-IL-18 into IL-1 $\beta$ and IL-18 $(37,38)$. Furthermore, LPS in the cytotype binds directly to mouse caspase- 11 or human caspase-4/5, which is a nonclassical pathway that activates the NLRP3 $(39,40)$.

Given the important role of neuroinflammation in the PD process and the significant anti-inflammatory effects of UTI, we speculate that UTI plays a neuroprotective role by inhibiting NLPR3. To test this hypothesis, we firstly identified the effects of different concentrations of UTI on cell viability and lactate dehydrogenase (LDH) release using PC12 and SH-SY5Y cells. The protective effect of UTI on $\mathrm{MPP}^{+}$treated cells was then assessed, and inflammatory factors were detected using ELISA. Finally, HPLC and western blot methods were used to detect dopamine (DA) 
secretion and tyrosine hydroxylase $(\mathrm{TH})$ protein expression. Through the above experiments, we expected to identify the neuroprotective effects of UTI in vitro and explore the underlying mechanisms.

We present the following article in accordance with the MDAR checklist (available at http://dx.doi.org/10.21037/ atm-21-1882).

\section{Methods}

The following materials were used: RPMI 1640 medium (Solarbio Life Sciences, Beijing, China), PC12 cell (Mlbio, m1052695, Shanghai, China), SH-SY5Y cells (Mlbio, ml052084, Shanghai, China), $\mathrm{MPP}^{+}$(SigmaAldrich, MO, USA), microplate reader (Thermo Fisher Scientific, MA, USA), LDH assay kit (Beyotime, Shanghai, China), ELISA kits (R\&D Systems, Minneapolis, USA), RIPA total protein lysate (Abcam, MA, USA), PVDF membranes (Thermo Fisher Scientific, USA), anti-TH (sc-25269, Santa Cruz Biotechnology, CA, USA), antiNLRP3 (SAB1410191, Sigma-Aldrich, USA), anticaspase-1 (PRS3459, Sigma-Aldrich, USA), anti-ASC (sc514414, Santa Cruz Biotechnology, USA), anti-IL-1 $\beta$ (sc-12742, Santa Cruz Biotechnology, USA), anti-IL-18 (PA5-79481, Thermo Fisher Scientific, USA), and antiGAPDH (G9545, Sigma-Aldrich, USA), HRP-conjugated secondary antibody (sc-2357, sc-2789, Santa Cruz Biotechnology, USA), chemiluminescent imaging system (Syngene, Cambridge, UK), HPLC (Agilent Technologies, CA, USA).

\section{Cell cultures and treatments}

The PC12 cells were cultured in RPMI 1640 medium with $5 \%$ fetal bovine serum (FBS). After incubation for 24 hours, nerve growth factor (NGF) at a concentration of $50 \mathrm{ng} / \mathrm{mL}$ was added to the medium to induce differentiation. After NGF addition for 4 days, subsequent experiments were performed. SH-SY5Y cells were cultured in DMEM including $1 \%$ streptomycin/penicillin as well as $10 \%$ FBS.

To detect whether UTI affects cell viability and LDH release, PC12 cells and SH-SY5Y cells were treated with different concentrations of UTI $(0,100,200,500$, $1,000 \mu \mathrm{M})$ for 6 hours, then the cell viability and LDH release were detected. To produce the experimental PD cell model, PC12 and SH-SY5Y cells were exposed to $100 \mu \mathrm{M} \mathrm{MPP}^{+}$ for 24 hours. Different concentrations of UTI were used to process cells before the PD cell model was established.

\section{Cell viability by the MTT cell assay}

Cell viability was detected by the MTT cell assay. Cells were seeded into 96-well plates and incubated under standard culture conditions for 72 hours. After UTI or $\mathrm{MPP}^{+}$treatment, the cells were incubated for 5 hours with MTT $(5 \mathrm{mg} / \mathrm{mL})$. The absorbance of individual wells was read at $570 \mathrm{~nm}$ using a microplate reader. Cell viability was determined by absorbance values.

\section{LDH release assay}

The LDH release assay was performed to assess the neuroprotective effects of UTI on PC12 or SH-SY5Y cells (41). Cells were seeded in 24-well plates at a concentration of $2 \times 10^{5}$ cells $/ \mathrm{mL}$. The amount of $\mathrm{LDH}$ was detected using the $\mathrm{LDH}$ assay kit according to the instructions of the manufacturer.

\section{Enzyme-linked immunosorbent assay (ELISA)}

The levels of interleukin-1 beta (IL-1 $\beta$ ), IL-6, and TNF- $\alpha$ were measured in PC12 and SH-SY5Y cell culture medium using ELISA kits according to the manufacturer's instructions (42). Optical density values were measured at $450 \mathrm{~mm}$ with a microplate reader in each well.

\section{Western blot assay}

Cellular proteins were extracted using RIPA total protein lysate, then separated by electrophoresis by SDS-PAGE and transferred onto PVDF. After incubation with blocking solution, the membranes were separately incubated with anti-TH, anti-NLRP3, anti-caspase-1, anti-ASC, anti-IL$1 \beta$, anti-IL-18, and anti-GAPDH at room temperature for 3 hours. Then, the HRP-conjugated secondary antibody was incubated with the blot for 1.5 hours. Image acquisition was performed by a chemiluminescent imaging system.

\section{High performance liquid chromatography (HPLC)}

The level of DA was measured by HPLC. After UTI or $\mathrm{MPP}^{+}$treatment, extracellular buffer was collected and stored at $-20^{\circ} \mathrm{C}$ until analysis. On the day of analysis, the samples were centrifuged at $10,000 \mathrm{~g}$ for $10 \mathrm{~min}$, and $3 \mu \mathrm{L}$ of supernatant was injected into the HPLC to measure DA. To quantify the sample peaks, each chemical species (DA) was compared to the external standards. 

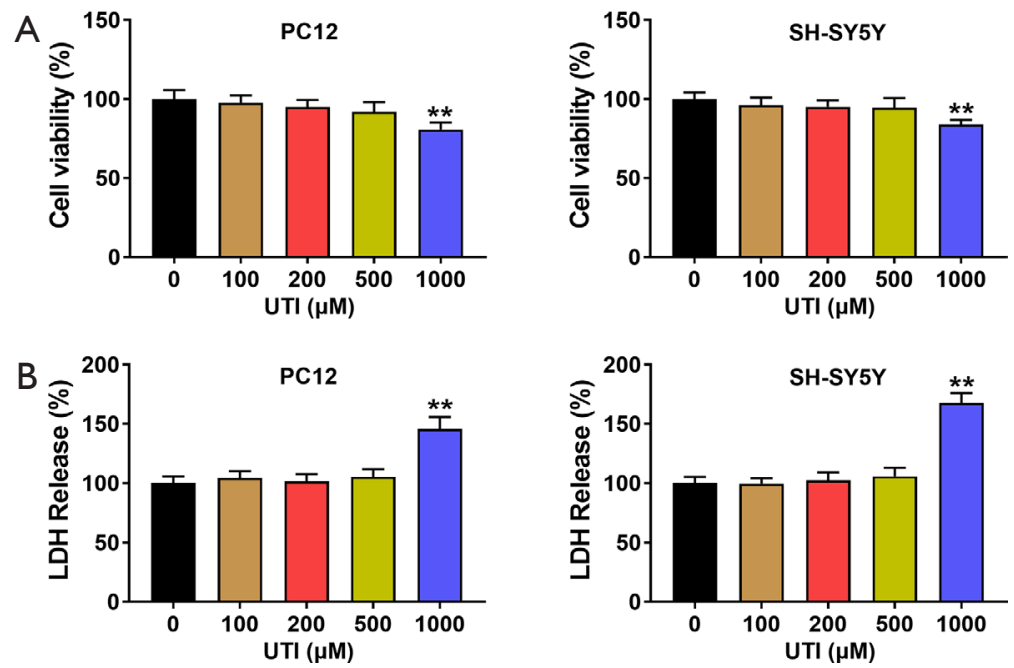

Figure 1 The effect of UTI on PC12 and SH-SY5Y cells. (A) The effect of different concentrations of UTI on cell viability. Concentrations of 0-500 $\mu$ M UTI had no significant effect on cell viability, while 1,000 $\mu$ M UTI significantly reduced the cell viability. (B) The effect of different concentrations of UTI on LDH release. Concentrations of 0-500 $\mu$ M UTI had no significant effect on LDH release, while 1,000 $\mu \mathrm{M}$ UTI significantly increased the LDH of PC12 and SH-SY5Y cells. Since a high concentration of UTI affected the viability and LDH level of PC12 cells and SH-SY5Y cells, 100, 200, and $500 \mu \mathrm{M}$ UTI treatments were used in subsequent experiments. **, P<0.01. UTI, ulinastatin; LDH, lactate dehydrogenase.

\section{Data processing and analysis}

Statistical analysis was performed using GraphPad Prism software (version 7.0). Differences between groups were analyzed using one-way analysis of variance (one-way ANOVA) followed by a Bonferroni post-hoc test. Data were considered significantly different when $\mathrm{P}<0.05$. All experiments in this study were performed in triplicate.

\section{Results}

\section{Low concentration of UTI has no significant effect on cell viability}

To verify whether different concentrations of UTI affected cell state, we first treated PC12 and SH-SY5Y cells with UTI at the concentrations of $100,200,500$, and $1,000 \mu \mathrm{M}$. After 6 hours of UTI treatment, cell viability was detected via the MTT cell assay, and LDH release was also detected. As shown in Figure 1, UTI treatment at concentrations of 100,200 , and $500 \mu \mathrm{M}$ did not significantly alter cell viability and $\mathrm{LDH}$ release compared to the control group (one-way ANOVA). Additionally, with 1,000 $\mu$ M UTI treatment, the cell viability of PC12 cells and SH-SY5Y cells decreased significantly $(\mathrm{P}<0.01)$ and $\mathrm{LDH}$ release increased significantly $(\mathrm{P}<0.01)$. These results indicated that low concentrations $(100,200,500 \mu \mathrm{M})$ of UTI had no significant effect on cell viability and did not affect LDH release levels. Since the high concentration of UTI affected the viability and LDH level of PC12 cells and SH-SY5Y cells, 100, 200, and $500 \mu \mathrm{M}$ UTI treatments were used in subsequent experiments.

\section{UTI increases the viability of MPP ${ }^{+}$treated cells and inhibits apoptosis}

In this study, PC12 and SH-SY5Y cells treated with $\mathrm{MPP}^{+}$at a concentration of $100 \mu \mathrm{M}$ were used as PD cell models. In order to assess the protective effect on cells, different concentrations of UTI were used to treat cells before modeling. As shown in Figure 2A, the MTT assay demonstrated that $\mathrm{MPP}^{+}$significantly reduced cell viability, while different concentrations of UTI pretreatment significantly enhanced the viability of $\mathrm{MPP}^{+}$ treated PC12 and SH-SY5Y cells (one-way ANOVA, $\mathrm{P}<0.01)$. Using the higher UTI concentration, the viability of the $\mathrm{MPP}^{+}$treated cells was closer to that of the control group. Correspondingly, as shown in Figure 2B, UTI pretreatment also significantly reversed the increase in $\mathrm{LDH}$ release caused by $\mathrm{MPP}^{+}$(one-way ANOVA, $\mathrm{P}<0.01$ ). Flow cytometric analysis showed that $\mathrm{MPP}^{+}$induced cell 

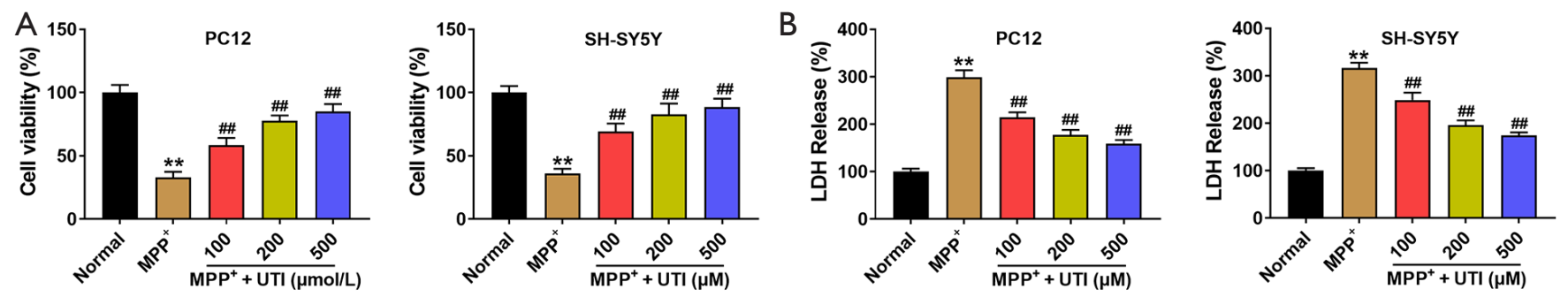
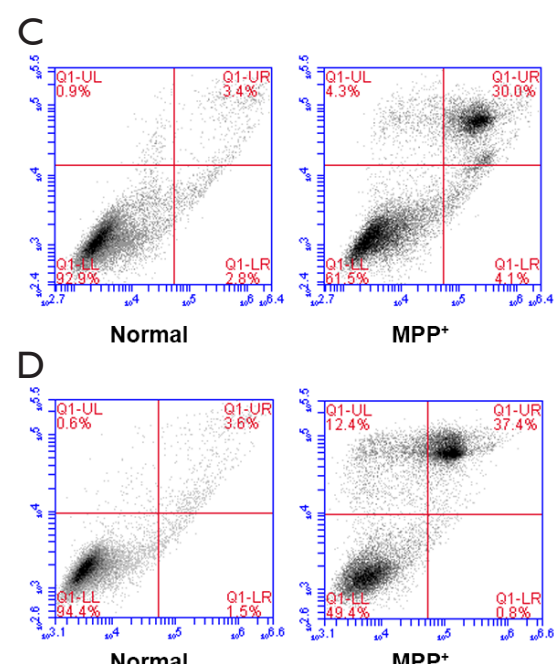

$\mathrm{MPP}^{+}$

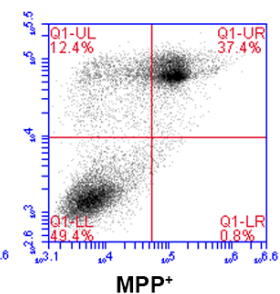

MPP $^{+}$

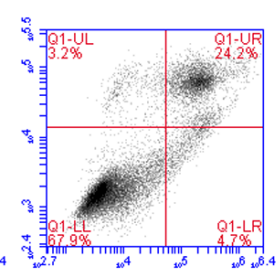

$\mathrm{MPP}^{+}+$UTI $100 \mu \mathrm{M}$

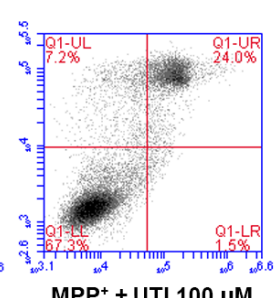

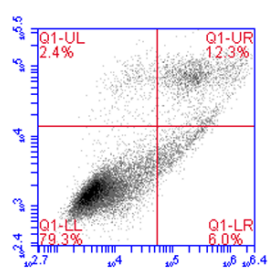

$\mathrm{MPP}^{+}+$UTI $200 \mu \mathrm{M}$

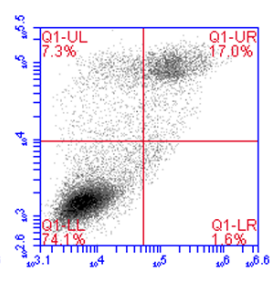

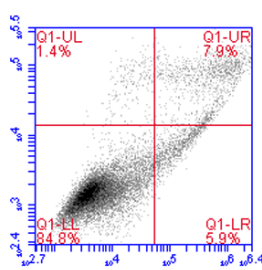

$\mathrm{MPP}^{+}+$UTI $500 \mu \mathrm{M}$

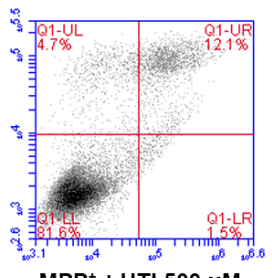

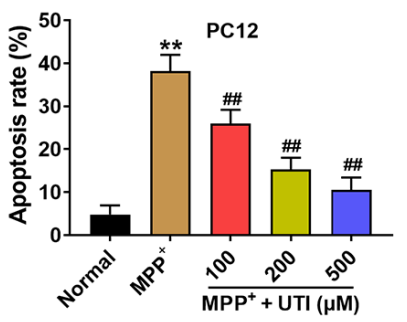

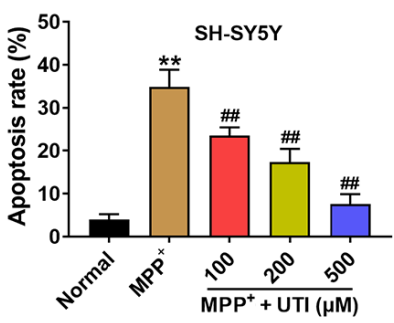

Figure 2 UTI enhances cell viability after $\mathrm{MPP}^{+}$treatment and inhibits apoptosis. (A) $\mathrm{MPP}^{+}$reduced $\mathrm{PC1} 2$ and $\mathrm{SH}-\mathrm{SY} 5 \mathrm{Y}$ cell viabilities, while UTI pre-treatment significantly enhanced PC12 and SH-SY5Y cell viabilities; (B) MPP ${ }^{+}$increased LDH release in PC12 and SHSY5Y cells, and UTI pre-treatment inhibited the release of LDH; (C,D) UTI pre-treatment significantly inhibited apoptosis of PC12 and SH-SY5Y cells induced by MPP ${ }^{+}$. The protective effect of UTI increased with the increase in concentration. ${ }^{* *}, \mathrm{P}<0.01 v s$. Normal group; \#\#, $\mathrm{P}<0.01$ vs. $\mathrm{MMP}^{+}$group. UTI, ulinastatin; $\mathrm{LDH}$, lactate dehydrogenase.

apoptosis, while UTI pre-treatment significantly reduced apoptosis, as shown in Figure $2 C, D$. It is worth noting that the protective effect of UTI was dose-dependent (one-way ANOVA, $\mathrm{P}<0.01)$. The dose dependence of the protective effect of UTI was also reflected in subsequent experiments.

\section{UTI inhibits the inflammatory response induced by $\mathrm{MPP}^{+}$}

$\mathrm{MPP}^{+}$induces apoptosis by activating inflammatory responses that stimulate cells to produce inflammatory factors. We used ELISA to detect inflammatory factors such as IL-1 $\beta$, IL-6, and TNF- $\alpha$ in PC12 cells and SH-SY5Y cells. For PC12 cells, compared to the control group, the levels of inflammatory factors increased significantly in the $\mathrm{MPP}^{+}$group, and UTI pre-treatment significantly reduced the levels of inflammatory factors (Figure 3, one-way ANOVA, $\mathrm{P}<0.01)$. For SH-SY5Y cells, UTI pre-treatment also showed similar anti-inflammatory effects (Figure 3, one-way ANOVA, $\mathrm{P}<0.01)$.

\section{UTI enhances DA release and TH expression in $\mathrm{MPP}^{+}$ treated cells}

In the above experiments, we demonstrated that UTI inhibited the inflammatory response induced by $\mathrm{MPP}^{+}$, thereby enhancing cell viability, inhibiting cell death, and playing a neuroprotective role. Another question was whether UTI protected the function of dopaminergic neurons. To explore this question, DA secretion levels were detected by HPLC and TH expression levels were detected via western blotting in PC12 and SH-SY5Y cells. As demonstrated in Figure 4A, in PC12 cells, $\mathrm{MPP}^{+}$ significantly reduced DA secretion, and UTI pre-treatment attenuated the inhibition of $\mathrm{MPP}^{+}$(one-way ANOVA, 

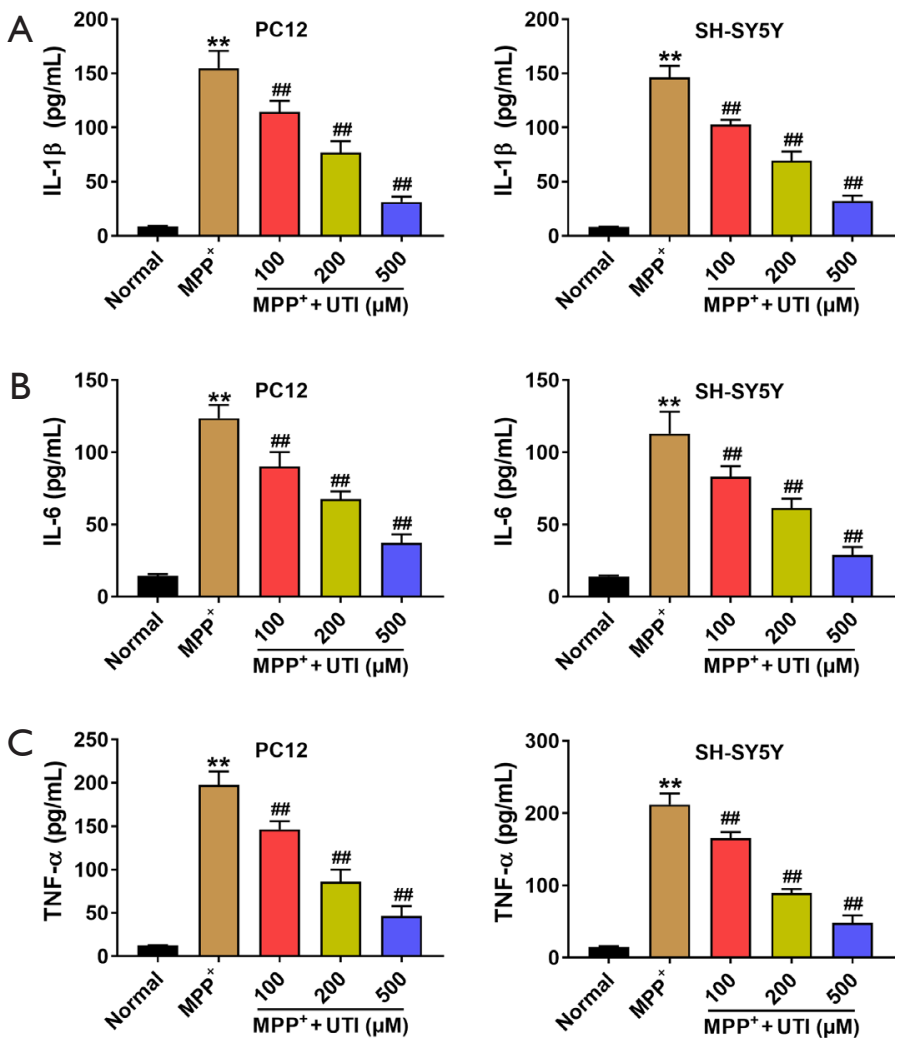

Figure 3 UTI inhibits the inflammatory response induced by $\mathrm{MPP}^{+}$. (A) $\mathrm{MPP}^{+}$significantly increased IL-1 $\beta$ levels of PC12 and SH-SY5Y cells, while UTI pre-treatment significantly reduced IL-1 $\beta$ levels in a concentration dependent manner; (B) MPP ${ }^{+}$increased the level of IL-6 in PC12 and SH-SY5Y cells, and UTI pre-treatment significantly reduced IL-6 levels in a concentration dependent manner; (C) UTI pretreatment reduced TNF- $\alpha$ levels that were increased by $\mathrm{MPP}^{+}$in $\mathrm{PC} 12$ and SH-SY5Y cells in a concentration dependent manner. **, $\mathrm{P}<0.01$ vs. Normal group; ${ }^{\#}, \mathrm{P}<0.01$ vs. $\mathrm{MPP}^{+}$group. UTI, ulinastatin.

$\mathrm{P}<0.01)$. The western blotting image of $\mathrm{TH}$ is on the left side of Figure $4 B$. In PC12 cells, $\mathrm{MPP}^{+}$significantly reduced TH expression and UTI pre-treatment also reversed the inhibition of $\mathrm{MPP}^{+}$(one-way ANOVA, $\mathrm{P}<0.05$ ). The same trend was found in SH-SY5Y cells, as shown in Figure $4 C, D$ (DA secretion test: one-way ANOVA, $\mathrm{P}<0.01$, TH expression test: one-way ANOVA, $\mathrm{P}<0.05)$.

\section{UTI inbibits the NLRP3 pathway}

To further explore the mechanism by which UTI inhibited $\mathrm{MPP}^{+}$induced inflammatory responses, we detected the protein levels of NLRP3 and related proteins by western blotting. As shown in Figure 5 A, in PC12 cells, $\mathrm{MPP}^{+}$ treatment significantly increased NLRP3, caspase-1, ASC, IL- $1 \alpha$, and IL-18 expression, and UTI pre-treatment reduced the over-expression of these proteins (one-way ANOVA, $\mathrm{P}<0.01)$. Similarly, in SH-SY5Y cells, MPP treatment significantly increased the expression levels of NLRP3, caspase-1, ASC, IL-1 $\alpha$, and IL-18, and UTI pretreatment reduced the over-expression of these proteins (Figure 5B; one-way ANOVA, $\mathrm{P}<0.01$ ). These results indicate that the anti-inflammatory effects exerted by UTI were achieved through inhibiting the NLRP3 pathway.

\section{Activation of NLRP3 eliminates the protective effect of UTI on MPP treated cells}

To confirm that UTI protected $\mathrm{MPP}^{+}$treated cells by inhibiting the activation of NLRP3, we detected the changes of cells after treated with NLRP3 activator Nigericin $(5 \mu M)$. As shown in Figure 6A, UTI pre-treatment $(500 \mu \mathrm{M})$ markedly decreased NLRP3, caspase-1, ASC, IL-1 $\alpha$, and IL-18 expression induced by $\mathrm{MPP}^{+}$treatment in PC12 and SH-SY5Y cells (one-way ANOVA, $\mathrm{P}<0.01$ ). Compared with $\mathrm{MPP}^{+}+$UTI group, Nigericin treatment significantly 

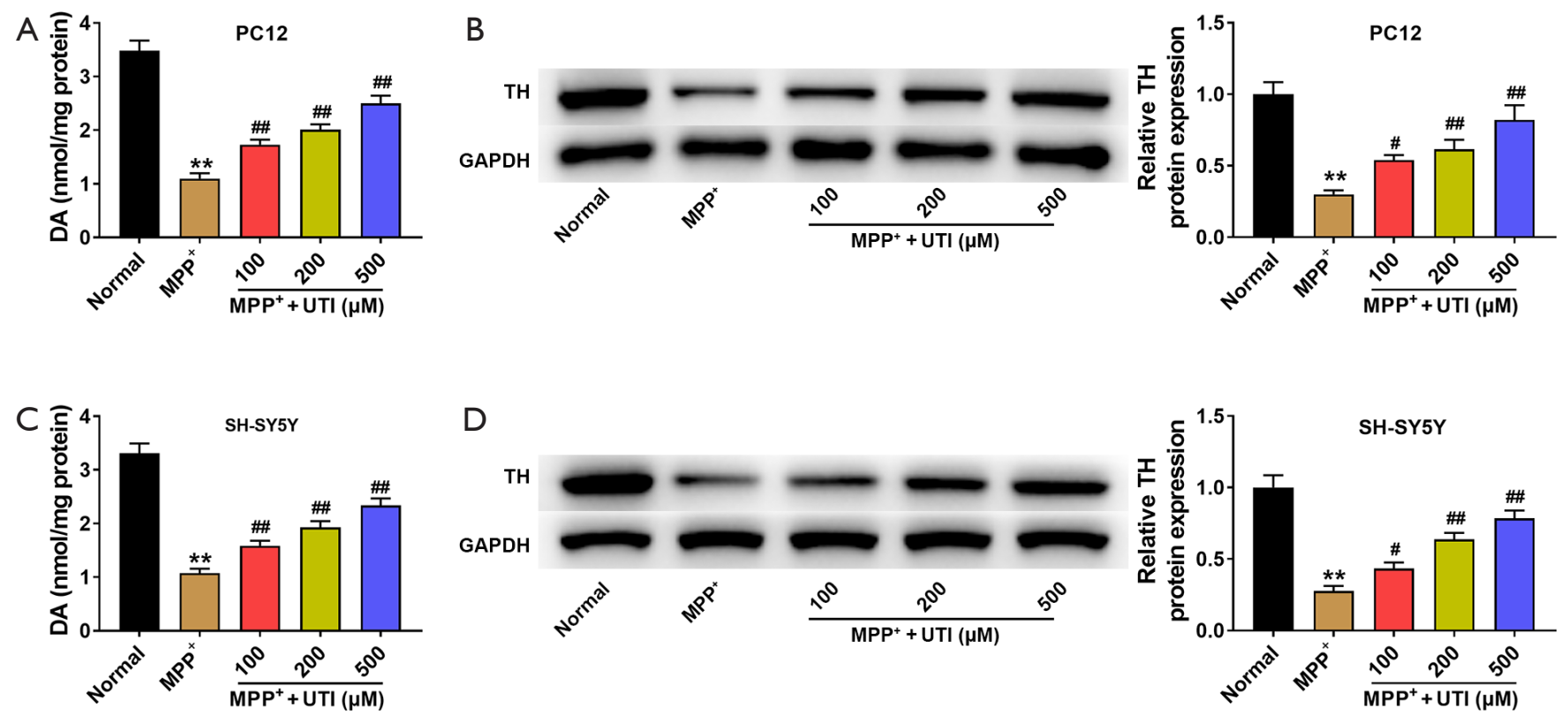

Figure 4 UTI increases the DA and TH levels of the PC12 and SH-SY5Y cells treated with MPP . $\left.^{+}, \mathrm{C}\right) \mathrm{MPP}^{+}$treatment significantly reduced the DA levels of PC12 cells and SH-SY5Y cells, while UTI pre-treatment attenuated DA levels in a concentration dependent manner; (B,D) $\mathrm{MPP}^{+}$treatment significantly inhibited TH expression in PC12 cells and SH-SY5Y cells, and UTI pre-treatment increased

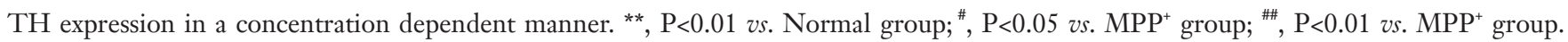
UTI, ulinastatin.
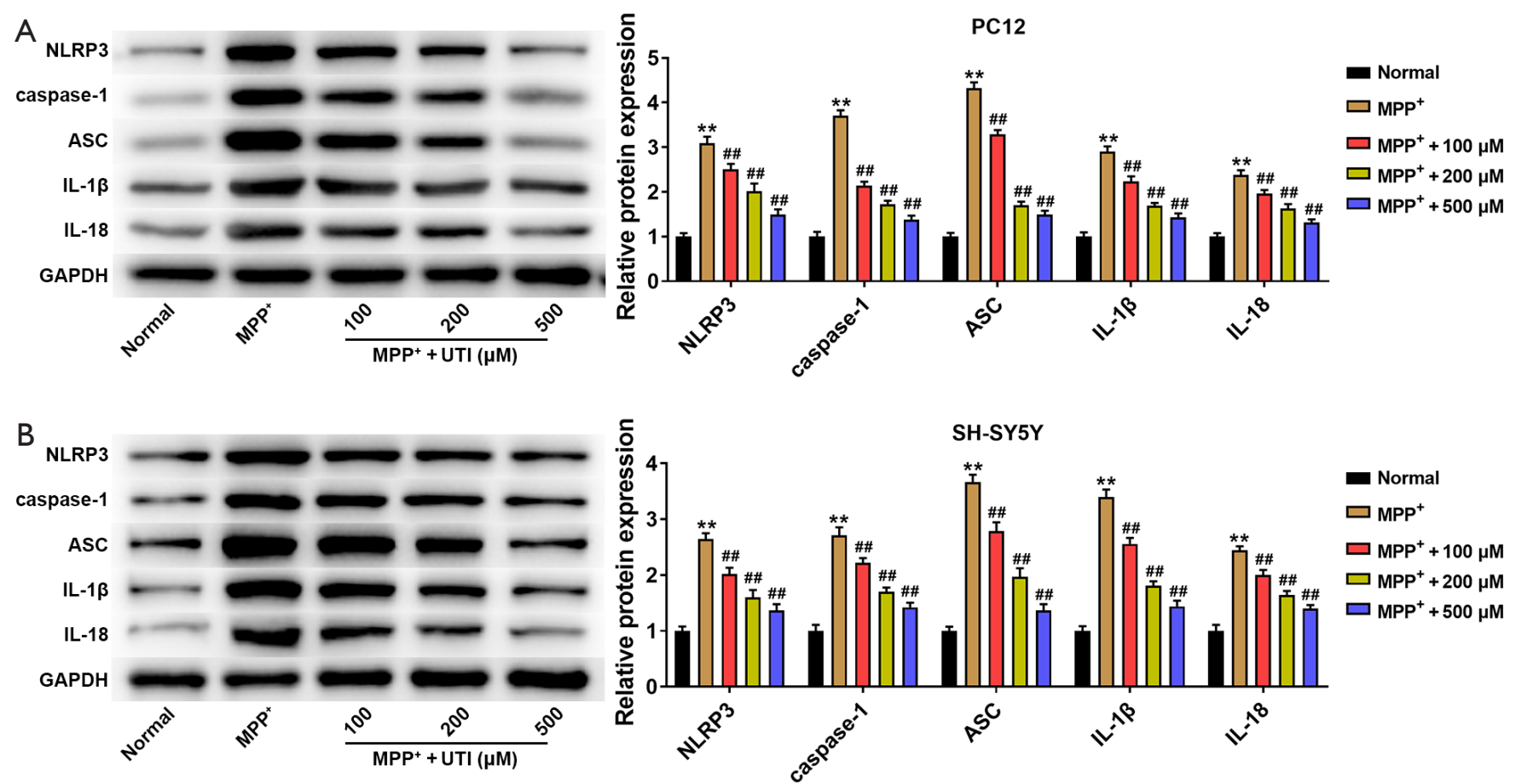

Figure 5 UTI inhibits the NLRP3 pathway. $\mathrm{MPP}^{+}$activated the NLRP3 pathway in PC12 cells (A) and SH-SY5Y cells (B), promoting NLRP3, caspase-1, ASC, IL-1 $\beta$, and IL-18 expression, while UTI pre-treatment significantly attenuated changes in the NRPL3 pathway. **, $\mathrm{P}<0.01$ vs. Normal group; ${ }^{\prime}, \mathrm{P}<0.01$ vs. $\mathrm{MPP}^{+}$group. UTI, ulinastatin; NLRP3, NOD-leucine rich repeat and pyrin containing protein 3. 


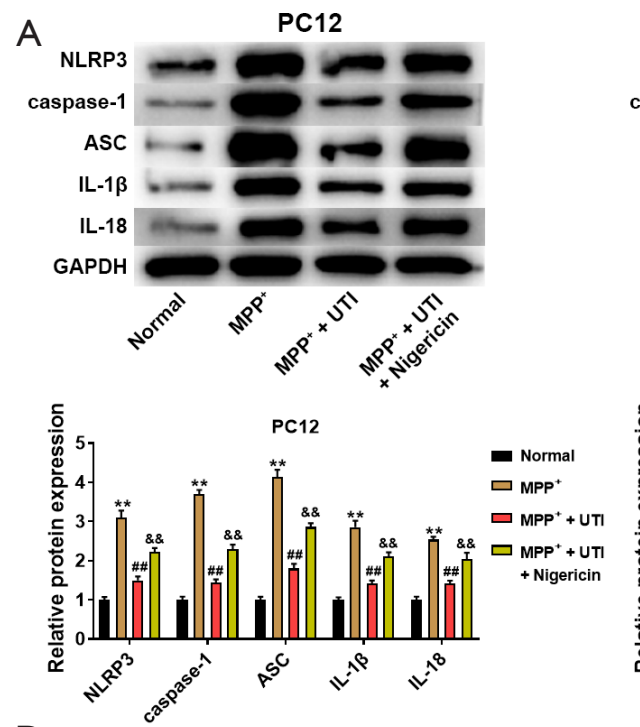

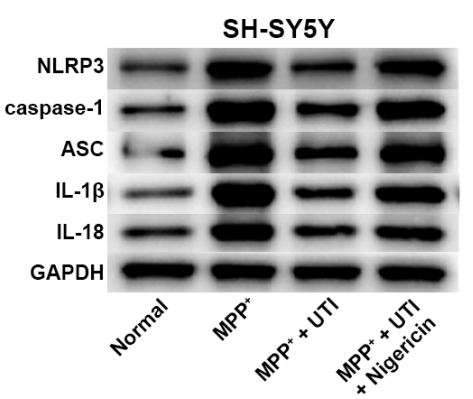

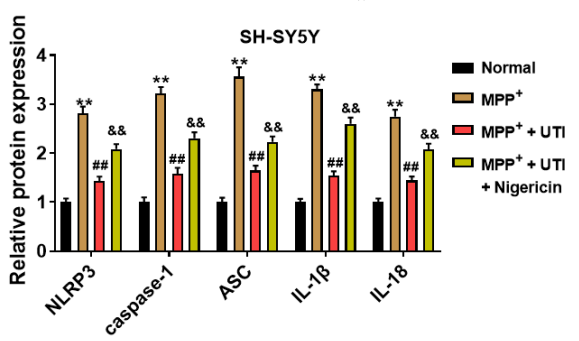

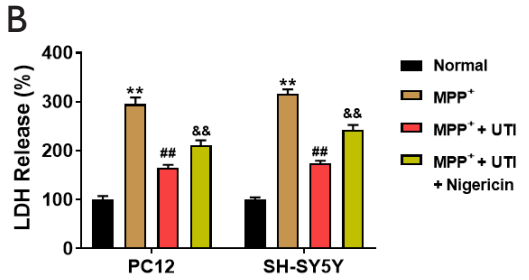

C

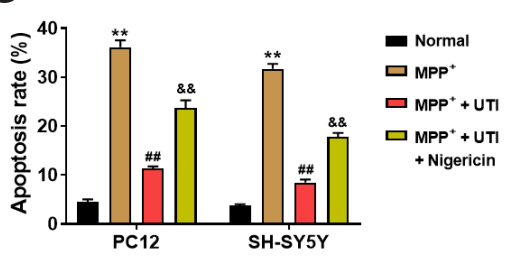

D
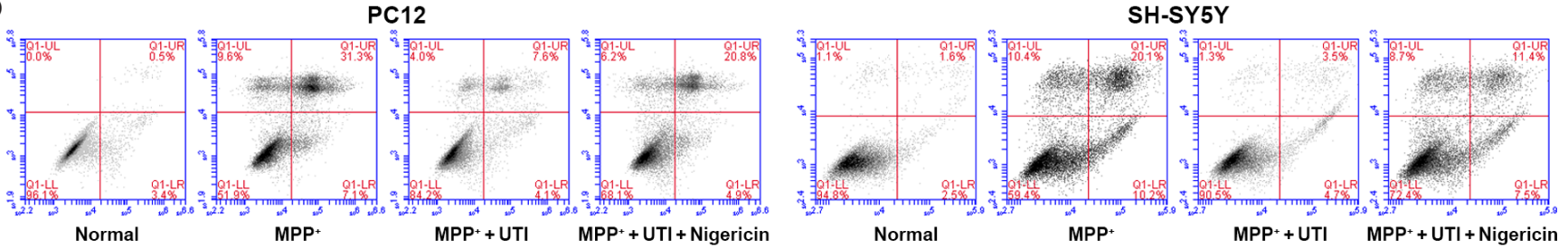

$$
\text { E ) }
$$
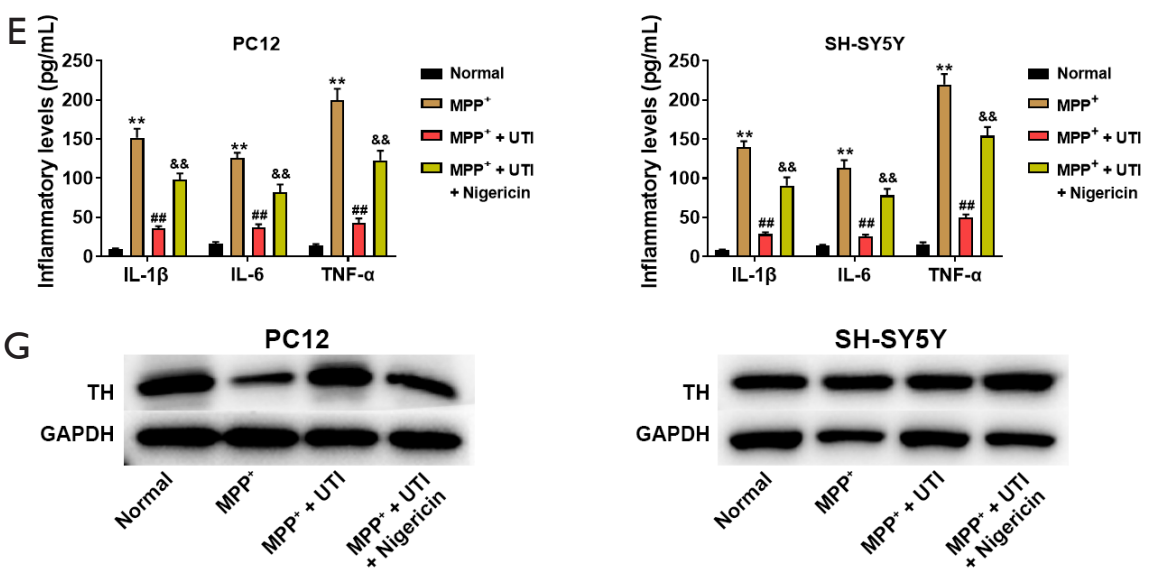

$\mathrm{F}$

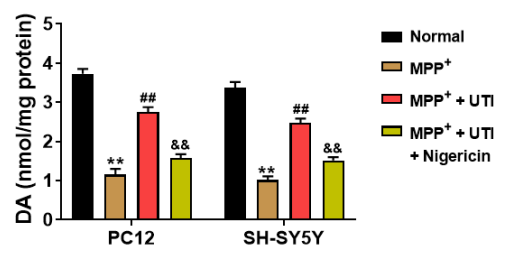

Figure 6 Activation of NLRP3 eliminates the protective effect of UTI on MPP ${ }^{+}$treated cells. (A) The NLRP3 activator Migericin significantly increased the protein expression of NLRP3, caspase-1, ASC, IL-1 $\beta$, and IL-18, and eliminated the inhibited effect of UTI on NLRP3 pathway in $\mathrm{MPP}^{+}$treated PC12 cells and SH-SY5Y cells. (B) Nigericin increased LDH release and eliminated the inhibited effect of UTI on LDH release in PC12 and SH-SY5Y cells. (C,D) Nigericin accelerated cell apoptosis eliminated the inhibited effect of UTI on apoptosis of PC12 and SH-SY5Y cells. (E) Nigericin significantly increased the levels of IL-1 $\beta$, IL-6, and TNF- $\alpha$, and eliminated the inhibited effect of UTI on inflammation of PC12 and SH-SY5Y cells. (F) Nigericin significantly increased DA levels and eliminated the promoting effect of UTI on DA of PC12 and SH-SY5Y cells. (G) Nigericin significantly increased TH protein expression and eliminated the promoting effect of UTI on TH protein of PC12 and SH-SY5Y cells. ${ }^{* *}, \mathrm{P}<0.01$ vs. Normal group; ${ }^{\# \#}, \mathrm{P}<0.01$ vs. MPP ${ }^{+}$group, ${ }^{\& \&}$, $\mathrm{P}<0.01$ vs. $\mathrm{MPP}^{+}+\mathrm{UTI}$ group. UTI, ulinastatin; LDH, lactate dehydrogenase; NLRP3, NOD-leucine rich repeat and pyrin containing protein 3. 
up-regulated NLRP3, caspase-1, ASC, IL-1 $\alpha$, and IL-18 expression (one-way ANOVA, $\mathrm{P}<0.01$ ). The results in Figure $6 B$ showed that Nigericin treatment significantly increased LDH release and eliminated the suppressed effect of UTI pre-treatment on LDH release (one-way ANOVA, $\mathrm{P}<0.01)$. Flow cytometric analysis showed that Nigericin treatment accelerated apoptosis and eliminated the inhibited effect of UTI on apoptosis of $\mathrm{MPP}^{+}$treated PC12 and SH-SY5Y cells (Figure $6 C$ and $D$, one-way ANOVA, $\mathrm{P}<0.01)$. Additionally, we detected inflammatory factors in PC12 cells and SH-SY5Y cells after Nigericin treatment. Compared to the $\mathrm{MPP}^{+}+\mathrm{UTI}$ group, the levels of IL$1 \beta$, IL-6, and TNF-asignificantly increased In the $\mathrm{MPP}^{+}$ + UTI + Nigericin group (Figure 6E, one-way ANOVA, $\mathrm{P}<0.01)$. As shown in Figure $6 F$ and $G$, Nigericin treatment significantly decreased the levels of DA secretion and $\mathrm{TH}$ expression in $\mathrm{MPP}^{+}+\mathrm{UTI}$ group, indicating that Nigericin eliminated the promoting effect of UTI on DA secretion and $\mathrm{TH}$ expression of $\mathrm{MPP}^{+}$treated PC12 and SH-SY5Y cells (one-way ANOVA, $\mathrm{P}<0.01$ ).

\section{Discussion}

In this study, we first treated cells with different concentrations of UTI to verify that low concentrations of UTI had no significant effect on cell viability. Subsequently, in the $\mathrm{MPP}^{+}$induced PD cell model, different concentrations of UTI pre-treatment were shown to enhance cell viability and inhibit apoptosis. Furthermore, we used ELISA to measure the levels of IL-1 $\beta$, IL-6, and TNF- $\alpha$, and the results showed that UTI pre-treatment significantly reduced the levels of inflammatory factors. On the other hand, to detect whether UTI could protect the function of neurons in the PD model, we used HPLC to detect DA secretion and western blot to detect cell TH expression, and found that UTI pre-treatment improved DA secretion and TH expression in PD model cells. To further explore the mechanism by which UTI inhibited $\mathrm{MPP}^{+}$induced inflammatory responses, we tested NLRP3 and related protein expression with western blotting. UTI was found to significantly reduce the expression of NLRP3, caspase-1, ASC, IL- $1 \alpha$, and IL-18 in $\mathrm{MPP}^{+}$treated cells. To confirm the role of NLRP3 in the protective mechanism of UTI on $\mathrm{MPP}^{+}$treated cells, the NLRP3 activator Nigericin was used to treat cells. The results showed that Nigericin treatment significantly increased the expression of NLRP3 related proteins, accelerated apoptosis, increased levels of inflammatory factors, and decreased DA secretion and
TH expression. These results showed that UTI played a neuroprotective role in PD model cells by inhibiting the NLRP3 pathway.

A previous study found that inflammatory cytokine IL-1 $\beta$ levels increased in the serum of patients with PD (28). Another group found that elevated levels of IL-1 $\beta$ in serum were mainly associated with the activation of NLRP3 in the brain and small glial cells in PD patients (43). The activation of NLRP3 promotes the maturation and secretion of IL- $1 \beta$ and increases neuroinflammation, thus promoting the development of PD (31,44-46). The main pathological changes in $\mathrm{PD}$ are the degeneration and disappearance of DA neurons in the substantia nigra, and neuroinflammation mediated by NLRP3 may lead to dopaminergic neuron degeneration in PD patients, aggravating the process of PD (47). A histological study from 2018 found an increase in NLRP3 expression in dopaminergic neurons in the brains of PD patients (48). These studies suggest that NLRP3 is a reasonable candidate target for PD.

As a protease inhibitor isolated from the urine of healthy adult males, UTI is safe to use and does not easily induce allergies. UTI reduces the inflammatory response through several processes such as anti-proteolysis, inhibition of inflammatory mediator release, and antioxidant reactions $(49,50)$. By activating the heme oxygenase 1 antioxidant pathway, UTI attenuates allergic inflammation (33). By reducing the translocation of lipopolysaccharide and systemic inflammation, UTI improves the cognitive function of elderly patients with spinal surgery (51). Besides, UTI could have a neuroprotective effect in the rat model of neuropathic pain $(52,53)$. Ulinastatin may attenuate the development of neuropathic pain when it was administered before the development of neuropathic pain (54). In our study, UTI pre-treatment inhibited the activation of NLRP3 and suppressed the expression of NLRP3 related proteins and the levels of inflammatory factors. We found that UTI significantly inhibited LDH release and cell apoptosis that induced by $\mathrm{MPP}^{+}$. Additionally, UTI increased DA secretion and TH expression of $\mathrm{MPP}^{+}$ treated PC12 and SH-SY5Y cells. However, after treated with Nigericin, a NLRP3 activator, the inhibited effect of UTI on the levels of inflammatory factors, LDH release, and cell apoptosis of $\mathrm{MPP}^{+}$treated cells were mostly eliminated. These results indicated that UTI performed a neuroprotective effect on $\mathrm{MPP}^{+}$induced PD cell models by inhibiting the activation of NLRP3.

One of the limitations of this study is the PD cell models 


\section{Page 10 of 12}

used in this study. It should be noted that at the tissue or overall level, neurons are in a more complex environment, and the neuroprotective effect of UTI needs to be further verified in PD animal models. Secondly, it is well known that the development and processes of $\mathrm{PD}$ are associated with the release of a variety of inflammatory factors. In this study, we focused only on the NLRP3 pathway. Therefore, suppressing these cytokines by inhibition of the NLRP3 pathway may be only one part of UTI's neuroprotective mechanism, and further research is needed to fully elucidate these mechanisms.

In conclusion, UTI significantly decreased LDH release, cell apoptosis and levels of inflammatory factors, improved cell viability and DA secretion. Its neuroprotective effect was achieved by inhibiting the NLRP3 pathway in the $\mathrm{MPP}^{+}$induced PD cell model.

\section{Acknowledgments}

Funding: None.

\section{Footnote}

Reporting Checklist: The authors have completed the MDAR checklist. Available at http://dx.doi.org/10.21037/atm-211882

Data Sharing Statement: Available at http://dx.doi. org/10.21037/atm-21-1882

Conflicts of Interest: All authors have completed the ICMJE uniform disclosure form (available at http://dx.doi. org/10.21037/atm-21-1882). The authors have no conflicts of interest to declare.

Ethical Statement: The authors are accountable for all aspects of the work in ensuring that questions related to the accuracy or integrity of any part of the work are appropriately investigated and resolved.

Open Access Statement: This is an Open Access article distributed in accordance with the Creative Commons Attribution-NonCommercial-NoDerivs 4.0 International License (CC BY-NC-ND 4.0), which permits the noncommercial replication and distribution of the article with the strict proviso that no changes or edits are made and the original work is properly cited (including links to both the formal publication through the relevant DOI and the license).
Lin et al. UTI inhibits NLRP3-induced apoptosis in a PD cell model

See: https://creativecommons.org/licenses/by-nc-nd/4.0/.

\section{References}

1. Guo M. Molecular pathways to Parkinson's disease. Mol Neurodegener 2012;7 Suppl 1:L13.

2. Shah RS, Chang SY, Min HK, et al. Deep Brain Stimulation: Technology at the Cutting Edge. J Clin Neurol 2010;6:167-82.

3. Dai HD, Otten B, Mehrkens JH, et al. A Novel Glove Monitoring System Used to Quantify Neurological Symptoms During Deep-Brain Stimulation Surgery. IEEE Sensors Journal 2013;13:3193-202.

4. Rissanen SM, Kankaanpaa M, Tarvainen MP, et al. Analysis of EMG and acceleration signals for quantifying the effects of deep brain stimulation in Parkinson's disease. IEEE Trans Biomed Eng 2011;58:2545-53.

5. Stacy M. Iconography: Medical Treatment of Parkinson Disease.

6. Giuffrida JP, Riley DE, Maddux BN, et al. Clinically deployable Kinesia technology for automated tremor assessment. Mov Disord 2009;24:723-30.

7. Jahanshahi M. Effects of deep brain stimulation of the subthalamic nucleus on inhibitory and executive control over prepotent responses in Parkinson's disease. Front Syst Neurosci 2013;7:118.

8. Zhou H, Shao M, Yang X, et al. Tetramethylpyrazine Analogue T-006 Exerts Neuroprotective Effects against 6-Hydroxydopamine-Induced Parkinson's Disease In Vitro and In Vivo. Oxid Med Cell Longev 2019;2019:8169125.

9. Hwang TY, Song MA, Ahn S, et al. Effects of Combined Treatment with Acupuncture and Chunggan Formula in a Mouse Model of Parkinson's Disease. Evid Based Complement Alternat Med 2019;2019:3612587.

10. Xu Q, Yan Q, Chen S. Ulinastatin is effective in reducing mortality for critically ill patients with sepsis: a causal mediation analysis. Sci Rep 2018;8:14360.

11. Del Pozzo-Magana BR, Lazo-Langner A, Carleton B, et al. A systematic review of treatment of drug-induced Stevens-Johnson syndrome and toxic epidermal necrolysis in children. J Popul Ther Clin Pharmacol 2011;18:e121.

12. Yao YT, Fang NX, Liu DH, et al. Ulinastatin reduces postoperative bleeding and red blood cell transfusion in patients undergoing cardiac surgery: A PRISMAcompliant systematic review and meta-analysis. Medicine 2020;99:e19184.

13. Zhou M, Lyu Y, Zhu Y, et al. Effect of Ulinastatin Combined With Dexmedetomidine on Postoperative 
Cognitive Dysfunction in Patients Who Underwent Cardiac Surgery. Front Neurol 2019;10:1293.

14. Wang J, Zhou J, Bai S. Combination of Glutamine and Ulinastatin Treatments Greatly Improves Sepsis Outcomes. J Inflamm Res 2020;13:109-15.

15. Liang S, Lai P, Li X, et al. Ulinastatin Reduces the Severity of Intestinal Damage in the Neonatal Rat Model of Necrotizing Enterocolitis. Med Sci Monit 2019;25:9123-30.

16. Li C, Ma D, Chen M, et al. Ulinastatin attenuates LPSinduced human endothelial cells oxidative damage through suppressing JNK/c-Jun signaling pathway. Biochem Biophys Res Commun 2016;474:572-8.

17. Wei F, Liu S, Luo L, et al. Anti-inflammatory mechanism of ulinastatin: Inhibiting the hyperpermeability of vascular endothelial cells induced by TNF- $\alpha$ via the RhoA/ROCK signal pathway. Int Immunopharmacol 2017;46:220-7.

18. Lotharius J, Brundin P. Pathogenesis of Parkinson's disease: dopamine, vesicles and alpha-synuclein. Nat Rev Neurosci 2002;3:932-42.

19. Wang Z, Luo XG, Gao C. Utility of susceptibilityweighted imaging in Parkinson's disease and atypical Parkinsonian disorders. Transl Neurodegener 2016;5:17.

20. Wang Q, Liu Y, Zhou J. Neuroinflammation in Parkinson's disease and its potential as therapeutic target. Transl Neurodegener 2015;4:19.

21. He Y, Hara H, Nunez G. Mechanism and Regulation of NLRP3 Inflammasome Activation. Trends Biochem Sci 2016;41:1012-21.

22. Davis BK, Wen H, Ting PY. The inflammasome NLRs in immunity, inflammation, and associated diseases. Annu Rev Immunol 2011;29:707-35.

23. Voet S, Srinivasan S, Lamkanfi M, et al. Inflammasomes in neuroinflammatory and neurodegenerative diseases. EMBO Mol Med 2019;11:e10248.

24. Duan Y, Kelley N, He Y. Role of the NLRP3 inflammasome in neurodegenerative diseases and therapeutic implications. Neural Regen Res 2020;15:1249-50.

25. Wang X, Chi J, Huang D, et al. $\alpha$-synuclein promotes progression of Parkinson's disease by upregulating autophagy signaling pathway to activate NLRP3 inflammasome. Exp Ther Med 2020;19:931-8.

26. Fan Z, Pan YT, Zhang ZY, et al. Systemic activation of NLRP3 inflammasome and plasma $\alpha$-synuclein levels are correlated with motor severity and progression in Parkinson's disease. J Neuroinflammation 2020;17:11.

27. Wang LY, Yu X, Li XX, et al. Catalpol Exerts a Neuroprotective Effect in the MPTP Mouse Model of
Parkinson's Disease. Front Aging Neurosci 2019;11:316.

28. Qiao C, Zhang Q, Jiang QL, et al. Inhibition of the hepatic Nlrp3 protects dopaminergic neurons via attenuating systemic inflammation in a MPTP/p mouse model of Parkinson's disease. J Neuroinflammation 2018;15:193.

29. Zhu J, Hu Z, Han X, et al. Dopamine D2 receptor restricts astrocytic NLRP3 inflammasome activation via enhancing the interaction of $\beta$-arrestin2 and NLRP3. Cell Death Differ 2018;25:2037-49.

30. Chhabra A, Ring AM, Weiskopf K, et al. Hematopoietic stem cell transplantation in immunocompetent hosts without radiation or chemotherapy. Sci Transl Med 2016;8:351ra105.

31. Bauernfeind FG, Horvath G, Stutz A, et al. Cutting edge: NF-kappaB activating pattern recognition and cytokine receptors license NLRP3 inflammasome activation by regulating NLRP3 expression. J Immunol 2009;183:787-91.

32. Liu W, Yin Y, Zhou Z, et al. OxLDL-induced IL-1 beta secretion promoting foam cells formation was mainly via CD36 mediated ROS production leading to NLRP3 inflammasome activation. Inflamm Res 2014;63:33-43.

33. Hussen J, Düvel A, Koy M, et al. Inflammasome activation in bovine monocytes by extracellular ATP does not require the purinergic receptor P2X7. Dev Comp Immunol 2012;38:312-20.

34. van Bruggen R, Köker MY, Jansen M, et al. Human NLRP3 inflammasome activation is Nox1-4 independent. Blood 2010;115:5398-400.

35. Zhou R, Tardivel A, Thorens B, et al. Thioredoxininteracting protein links oxidative stress to inflammasome activation. Nat Immunol 2010;11:136-40.

36. Misawa T, Takahama M, Kozaki T, et al. Microtubuledriven spatial arrangement of mitochondria promotes activation of the NLRP3 inflammasome. Nat Immunol 2013;14:454-60.

37. Lamkanfi M, Dixit VM. Mechanisms and functions of inflammasomes. Cell 2014;157:1013-22.

38. Vanaja SK, Rathinam VAK, Fitzgerald KA. Mechanisms of inflammasome activation: recent advances and novel insights. Trends Cell Biol 2015;25:308-15.

39. de Carvalho RVH, Andrade WA, Lima-Junior DS, et al. Leishmania Lipophosphoglycan Triggers Caspase-11 and the Non-canonical Activation of the NLRP3 Inflammasome. Cell Rep 2019;26:429-37.e5.

40. Baker PJ, Boucher D, Bierschenk D, et al. NLRP3 inflammasome activation downstream of cytoplasmic LPS recognition by both caspase-4 and caspase-5. Eur J Immunol 2015;45:2918-26. 


\section{Page 12 of 12}

41. Wu L, Jiang C, Kang Y, et al. Curcumin exerts protective effects against hypoxia-reoxygenation injury via the enhancement of apurinic/apyrimidinic endonuclease 1 in SH-SY5Y cells: Involvement of the PI3K/AKT pathway. Int J Mol Med 2020;45:993-1004.

42. Xu J, Sun Y, Lu J. Knockdown of Long Noncoding RNA (lncRNA) AK094457 Relieved Angiotensin II Induced Vascular Endothelial Cell Injury. Med Sci Monit 2020;26:e919854.

43. Dinarello CA, Simon A, van der Meer JW. Treating inflammation by blocking interleukin-1 in a broad spectrum of diseases. Nat Rev Drug Discov 2012;11:633-52.

44. Próchnicki T, Mangan MS, Latz E. Recent insights into the molecular mechanisms of the NLRP3 inflammasome activation. F1000Res 2016;5:1469.

45. Burre J, Sharma M, Sudhof TC. Definition of a Molecular Pathway Mediating $\alpha$-Synuclein Neurotoxicity. J Neurosci 2015;35:5221-32.

46. Codolo G, Plotegher N, Pozzobon T, et al. Triggering of Inflammasome by Aggregated $\alpha$-Synuclein, an Inflammatory Response in Synucleinopathies. PLoS One 2013;8:e55375.

47. Liu Y, Fiskum G, Schubert D. Generation of reactive oxygen species by the mitochondrial electron transport chain. J Neurochem 2002;80:780-7.

48. Verma D, Lerm M, Blomgran Julinder R, et al. Gene polymorphisms in the NALP3 inflammasome are

Cite this article as: Lin $\mathrm{Y}, \mathrm{Xu} \mathrm{D}$, Gao F, Zheng X. Ulinastatin inhibits NLRP3-induced apoptosis in a PD cell model. Ann Transl Med 2021;9(11):924. doi: 10.21037/atm-21-1882
Lin et al. UTI inhibits NLRP3-induced apoptosis in a PD cell model

associated with interleukin-1 production and severe inflammation: relation to common inflammatory diseases? Arthritis Rheum 2008;58:888-94.

49. Lv ZT, Huang JM, Zhang JM, et al. Effect of Ulinastatin in the Treatment of Postperative Cognitive Dysfunction: Review of Current Literature. Biomed Res Int 2016;2016:2571080.

50. Song D, Song G, Niu Y, et al. Ulinastatin Activates Heme Oxygenase-1 Antioxidant Pathway and Attenuates Allergic Inflammation. Br J Pharmacol 2014;171:4399-412.

51. Zhang M, Zhang YH, Fu HQ, et al. Ulinastatin May Significantly Improve Postoperative Cognitive Function of Elderly Patients Undergoing Spinal Surgery by Reducing the Translocation of Lipopolysaccharide and Systemic Inflammation. Front Pharmacol 2018;9:1007.

52. Jung KT, Lee HY, Yoon MH, et al. The effect of urinary trypsin inhibitor against neuropathic pain in rat models. Korean J Pain 2013;26:356-60.

53. Ouyang H, Nie B, Wang $\mathrm{P}$, et al. Ulinastatin attenuates neuropathic pain induced by L5-VRT via the calcineurin/ IL-10 pathway. Mol Pain 2016;12:1744806916646785.

54. Oh SH, So HJ, Lee HY, et al. Urinary trypsin inhibitor attenuates the development of neuropathic pain following spinal nerve ligation. Neurosci Lett 2015;590:150-5.

(English Language Editor: C. Betlazar-Maseh) 